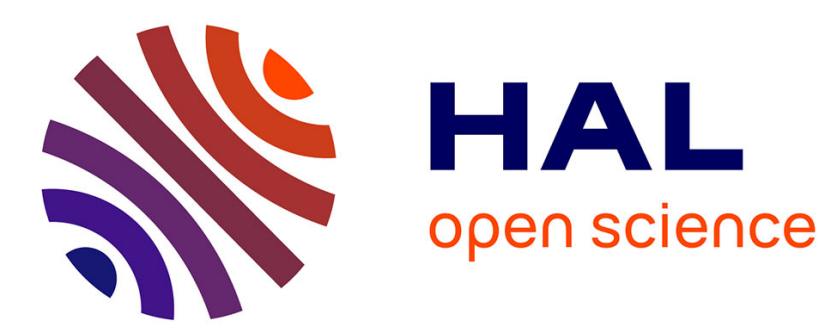

\title{
Stabilization of switched affine systems with disturbed state-dependent switching laws
}

\author{
Zohra Kader, Christophe Fiter, Laurentiu Hetel, Lotfi Belkoura
}

\section{To cite this version:}

Zohra Kader, Christophe Fiter, Laurentiu Hetel, Lotfi Belkoura. Stabilization of switched affine systems with disturbed state-dependent switching laws. International Journal of Robust and Nonlinear Control, 2018, 28 (2), pp.582-595. 10.1002/rnc.3887 . hal-01689247

\section{HAL Id: hal-01689247 \\ https://hal.inria.fr/hal-01689247}

Submitted on 23 Feb 2018

HAL is a multi-disciplinary open access archive for the deposit and dissemination of scientific research documents, whether they are published or not. The documents may come from teaching and research institutions in France or abroad, or from public or private research centers.
L'archive ouverte pluridisciplinaire HAL, est destinée au dépôt et à la diffusion de documents scientifiques de niveau recherche, publiés ou non, émanant des établissements d'enseignement et de recherche français ou étrangers, des laboratoires publics ou privés. 


\title{
Stabilization of switched affine systems with disturbed state-dependent switching laws
}

\author{
Zohra Kader ${ }^{1,2}$, Christophe Fiter ${ }^{1}$, Laurentiu Hetel ${ }^{3}$ and Lotfi Belkoura ${ }^{1,2}$.
}

\begin{abstract}
In this paper we investigate the stabilization problem for a class of switched affine systems with a state-dependent switching law. Since the states measurements are in general subject to perturbations and noises, we propose a robust switching law design method. Qualitative conditions for the stability of the closed-loop switched system are given. Stability conditions are also formulated as Linear Matrix Inequalities (LMIs) in order to allow numerical implementations. Results are illustrated by numerical examples in order to show the efficiency of the method and its limits.
\end{abstract}

\section{Index Terms}

Switched affine systems, switching control, bilinear systems, $R \epsilon$-stability, robust stabilization Switched affine systems, switching control, bilinear systems, $R \epsilon$-stability, robust stabilization

\section{INTRODUCTION}

Switched systems represent a popular class of hybrid systems [2], [9], [19], [31], [32]. In the present work we are interested in the stabilization problem of switched affine systems with a state dependent switching law (see for instance [7], [13], [23]). This class of systems has attracted interest of the control community since its study has direct applications in power electronics [13], [25], [42] (for example DC/DC and AC/DC converters). However, the control of switched affine systems presents some difficulties: fast switching, presence of non standard equilibrium points (when different subsystems do not share a common equilibrium), sliding dynamics, etc. Furthermore, for such systems it is required to use more general concepts of solutions than the classical one in order to take into account the dynamics over the discontinuities [15].

In the literature, we can find elegant design methods based on the existence of a stable convex combination [7], [13], [18]. Using multiple Lyapunov functions, a switching law is designed in [45]. This method was extended to the case of uncertain equilibria in [46]. In [47], $\mathcal{H}_{\infty}$ performances are guaranteed by the design methodology. Numerical approaches have been provided in other papers, based on Model Predictive Control (MPC) [5] and optimal controllers (see for instance [6], [34], [35], [39], [41], [22]). Particular applications of sliding mode control to switched systems can be found in [42]. Results on switched systems with delayed state-dependent switching law design are also considered in [16]. Recently, a constructive method for the design of state-dependent switching laws has been proposed in [23]. This method uses a bilinear model [14], [17] of the system and the existence of a stabilizing continuous control in order to derive a stabilizing switching law.

The present paper provides a robust design method of a state-dependent switching law for systems with perturbations. The main advantages of the results presented here with respect to the existing works in the literature lie in the following two aspects:

1) In this paper, the classical condition concerning the existence of a Hurwitz convex combination is avoided. Compared to the approaches in [7], [13], [18] the proposed methodology can be interpreted as an approach that uses state-dependent convex combinations.

2) To the best of our knowledge, the approaches in [7], [13], [18], [23], [24], consider that the states measurements used in the switching laws are always free of perturbations and noises. However, this is rarely the case in practical applications and dealing with such a perturbation is a challenging problem. In the present paper, we consider that the state is affected by a bounded perturbation, and provide a robust state-dependent switching law design method to ensure the stabilization of switched affine systems.

We start directly with the bilinear system formulation proposed in [23]. Both qualitative and numerical conditions of stability are developed. The study can also be related with LMI design methodology proposed in [1], [43], [44]. The efficiency of the method is illustrated by simulation results.

This paper is organized as follows. In Section II, the problem under study is stated. In Section III, the control design idea is expressed and the analytic result is given. An LMI approach is given in Section IV. The results are illustrated by numerical examples in Section V. The paper is ended with a conclusion and perspectives.

*This work was supported by the Agence Nationale pour la Recherche Project ROCC-SYS under the Grant agreement ANR-14-C27-0008 and by the ARCIR Project ESTIREZ.

Zohra $\operatorname{Kader}^{1,2}$ is with CRIStAL CNRS UMR 9189, Université Lille 1, Sciences et Technologies, Villeneuve d'Ascq, France. She is also with Non-A, INRIA - Lille Nord Europe, Villeneuve d'Ascq, France. zohra.kader@inria.fr.

Fiter Christophe ${ }^{1}$ is with CRIStAL CNRS UMR 9189, Université Lille 1, Sciences et Technologies, Villeneuve d'Ascq, France Christophe.Fitereuniv-lillel.fr.

Hetel Laurentiu ${ }^{3}$ is with CRIStAL CNRS UMR 9189, École Centrale de Lille, Villeneuve d'Ascq, France laurentiu.hetel@ec-lille.fr.

Belkoura Lotf ${ }^{1,2}$ is with CRIStAL CNRS UMR 9189, Université Lille 1, Sciences et Technologies, Villeneuve d'Ascq, France. He is also with Non-A, INRIA - Lille Nord Europe, Villeneuve d'Ascq, France Lotfi.Belkoura@univ-lillel.fr

${ }^{2}$ This work was supported by the Agence Nationale pour la Recherche Project ROCC-SYS under the Grant ANR-14-C27-0008. 


\section{A. Notations}

In this paper we use the notation $\mathbb{R}_{+}$to refer to the interval $[0, \infty)$. The transpose of a matrix $M$ is denoted by $M^{T}$. In a symmetric matrix the elements that can be deduced by symmetry are denoted by $*$. The notation $M \succeq 0$ (resp. $M \preceq 0$ ) means that the matrix $M$ is positive (resp. negative) semi-definite, and the notation $M \succ 0$ (resp. $M \prec 0$ ) means that it is positive definite (resp. negative definite). The identity matrix is denoted by $I$. Notations $\lambda_{\min }(M)$ and $\lambda_{\max }(M)$ are used to refer to the minimum and maximum eigenvalue respectively of a square matrix $M$. The notation $M_{(i)}$ refers to the $i$-th row of a matrix (or vector). For a positive definite matrix $P \in \mathbb{R}^{n \times n}$ and a positive scalar $\gamma$, we denote by $\mathcal{E}(P, \gamma)$ the ellipsoid

$$
\mathcal{E}(P, \gamma)=\left\{x \in \mathbb{R}^{n}: x^{T} P x \leq \gamma\right\} .
$$

For all positive scalar $r$, we denote by $\mathcal{B}(0, r)$ the ball of radius $\sqrt{r}$ :

$$
\mathcal{B}(0, r)=\mathcal{E}(I, r)=\left\{x \in \mathbb{R}^{n}: x^{T} x \leq r\right\}
$$

For a given set $\mathcal{S}$, the notation $\operatorname{Conv}\{\mathcal{S}\}$ indicates the convex hull of the set, $\operatorname{int}\{\mathcal{S}\}$ its interior and $\overline{\mathcal{S}}$ its closure. Finally, the closed convex hull of the set $\mathcal{S}$ will be noted by $\overline{\operatorname{Conv}}\{\mathcal{S}\}$. Let $\mathcal{S} \subset \mathbb{R}^{m}$ be a finite set of vectors. The minimum argument of a given function $f: \mathcal{S} \longrightarrow \mathbb{R}$ is noted by

$$
\underset{x \in \mathcal{S}}{\arg \min } f(x)=\{y \in \mathcal{S}: f(y) \leq f(z), \forall z \in \mathcal{S}\}
$$

For a positive integer $N$, we denote by $\mathcal{I}_{N}$ the set $\{1, \ldots, N\}$. We use $\|\cdot\|$ to denote the Euclidean norm for a vector and the associated norm for a matrix. By $\Delta_{N}$ we denote the unit simplex

$$
\Delta_{N}=\left\{\beta=\left[\beta_{(1)}, \quad \ldots, \quad \beta_{(N)}\right]^{T} \in \mathbb{R}^{N}: \sum_{i=1}^{N} \beta_{(i)}=1, \beta_{(i)} \geq 0, i \in \mathcal{I}_{N}\right\} .
$$

\section{PRELIMINARIES AND PROBLEM STATEMENT}

\section{A. System description}

Consider the following system

$$
\dot{x}=A x+\sum_{i=1}^{m}\left(N_{i} x+b_{i}\right) u_{(i)},
$$

with $x \in \mathbb{R}^{n}$ and $u_{(i)}$ the $i$-th component of the input $u$. The input $u$ is only allowed to take values in the set $\mathcal{V}=$ $\left\{v_{1}, \ldots, v_{N}\right\} \subset \mathbb{R}^{m} . A \in \mathbb{R}^{n \times n}, B=\left[b_{1}, \ldots, \quad b_{m}\right] \in \mathbb{R}^{n \times m}$, and $N_{i} \in \mathbb{R}^{n \times n}$ are the matrices describing the system. system

It has been demonstrated in [23] that the class of switched system (1) is quite general in the sense that any switched affine

$$
\begin{aligned}
& \dot{x}=\tilde{A}_{\sigma} x+\tilde{b}_{\sigma}, \\
& \sigma \in \mathcal{I}_{N},
\end{aligned}
$$

with $\tilde{A}_{i} \in \mathbb{R}^{n \times n}$ and $\tilde{b}_{i} \in \mathbb{R}^{n \times 1}$ the matrices describing the subsystems, can be represented in the form (1) (see Proposition 1 in [23]).

Note that, if the bilinear terms of system (1) are null, the system can model a linear system with a relay feedback controller which is widely studied in the literature (see for instance [20], [21], [27], [28], [33]).

In the sequel we assume that:

A-1 The pair $(A, B)$ is controllable, which implies that there exists a matrix $K$ such that the closed-loop matrix $A_{c l}=A+B K$ is Hurwitz.

A-2 The set $\operatorname{int}\{\operatorname{Conv}\{\mathcal{V}\}\}$ is nonempty, and the null vector is contained inside $(0 \in \operatorname{int}\{\operatorname{Conv}\{\mathcal{V}\}\})$.

This paper deals with the stabilization of system (1) in the case of a disturbed switching law. We consider a controller inspired from the min-switching strategies in [12], [26], [36] given by

$$
u(x+e(t)) \in \underset{v \in \mathcal{V}}{\arg \min }(x+e(t))^{T} \Gamma v
$$

where $e$ is an exogenous unknown disturbance considered as a measurable and bounded function from $\mathbb{R}_{+}$to $\mathbb{R}^{n}$ satisfying

$$
e(t)^{T} e(t) \leq \bar{e}
$$

with $\bar{e}$ its upper bound. The matrix $\Gamma \in \mathbb{R}^{n \times m}$ characterizes the switching hyperplanes of the control and will take a particular form, in the sequel, obtained using the Lyapunov theory. The formulation of the controller (3) encompasses the classical sign function in the relay feedback. Indeed, if $\mathcal{V}=\left\{v_{1}, v_{2}\right\}=\{-v, v\} \subset \mathbb{R}$ with $v>0$ then we get

$$
u(x+e(t)) \in-v \operatorname{sign}\left(\Gamma^{T}(x+e(t))=\left\{\begin{array}{l}
\{v\} \quad \text { if } \Gamma^{T}(x+e(t))<0, \\
\{-v, v\} \text { if } \Gamma^{T}(x+e(t))=0, \\
\{-v\} \text { if } \Gamma^{T}(x+e(t))>0 .
\end{array}\right.\right.
$$

Thus the closed-loop system is modeled by a differential equation with discontinuous right hand-side [10]. Consequently, in order to study the stability of the system we will consider the Filippov solutions [15] based on the use of differential inclusions [3]. 


\section{B. Solution concept}

The interconnection (1), (3) is the closed-loop system modeled by a discontinuous differential equation of the form

$$
\dot{x}=A x+\sum_{i=1}^{m}\left(N_{i} x+b_{i}\right) \tilde{u}_{(i)}(t, x)=f(t, x),
$$

where $\tilde{u}_{(i)}(t, x)$ is the $i$-th component of $\tilde{u}(t, x)=\left[\begin{array}{c}\tilde{u}_{(1)}(t, x) \\ \vdots \\ \tilde{u}_{(m)}(t, x)\end{array}\right]=u(x+e(t))$.

To the discontinuous closed-loop system (6) we associate the differential inclusion

$$
\dot{x} \in \mathcal{F}(t, x),
$$

with $\mathcal{F}(t, x)$ a set-valued map which can be computed using the construction given in [4], [15] such that

$$
\mathcal{F}(t, x)=\bigcap_{\delta>0} \bigcap_{\mu(\mathcal{S})=0} \overline{\operatorname{Conv}}\{f(t, \breve{\mathcal{B}}(x, \delta) \backslash \mathcal{S})\}, \forall x \in \mathbb{R}^{n}, t \in \mathbb{R}_{+},
$$

where $\overline{\text { Conv }}$ is the closed convex hull, $\breve{\mathcal{B}}(x, \delta)$ is the open ball centered on $x$ with radius $\sqrt{\delta}$, and $\mathcal{S} \subset \mathbb{R}^{n}$ with $\mu(\mathcal{S})$ its measure in the sense of Lebesgue. Hereafter, we call $\mathcal{F}(t, x)$ the set-valued map associated to the discontinuous system (6). (6).

The closed-loop system is then modeled by the differential inclusion (7) which can be used to define solutions for system

Definition 1 (Filippov solution): Consider the closed-loop system (6) and its associated differential inclusion (7). A Filippov solution of the discontinuous systems (6) over the interval $\left[t_{a}, t_{b}\right] \subset[0, \infty)$ is an absolutely continuous mapping $y:\left[t_{a}, t_{b}\right] \longrightarrow$ $\mathbb{R}^{n}$ satisfying:

$$
\dot{y}(t) \in \mathcal{F}(t, y(t)), \quad \text { for almost all } t \in\left[t_{a}, t_{b}\right],
$$

with $\mathcal{F}(t, x)$ given by (8).

A differential inclusion has at least one solution if the set valued map $\mathcal{F}(t, x)$ is locally bounded and takes nonempty, compact and convex values [4], [10]. Over the article the following notion of stability will be used.

Definition 2 (R $\epsilon$-stability): Consider positive scalars $R$ and $\epsilon$, such that $\epsilon<R$. Assume that there exists a matrix $P=$ $P^{T} \succ 0$ such that for all Filippov solutions $x($.$) of system (6) with x(0) \in \mathcal{E}(P, R)$, the value of the state $x(t)$ converges to $\mathcal{E}(P, \epsilon)$ as $t$ goes to infinity. Then, system (6) is said to be $R \epsilon$-stable from $\mathcal{E}(P, R)$ to $\mathcal{E}(P, \epsilon)$.

This notion of stability is adapted from [37], where it was used for relay systems with input delays.

The closed-loop system (1), (3), (4) is affected by a disturbance in the actuator. In this paper, for bounded disturbances, we give conditions under which the system is $R \epsilon$-stable. Sufficient LMIs stability conditions are also given.

\section{CONTROL DESIGN}

This section deals with the $R \epsilon$-stabilization of system (1) under bounded input disturbances. Assumptions A.1 and A.2 are used to prove that there exists a switching control law such that the system is $R \epsilon$-stable.

Theorem 1: Assume that A.1 and A.2 hold. Then there exist positive scalars $R, \epsilon$, a matrix $P=P^{T} \succ 0$, and a switching control law $u(x+e(t))$ such that system (1) is $R \epsilon$-stable from $\mathcal{E}(P, R)$ to $\mathcal{E}(P, \epsilon)$, for an input perturbation $e$ satisfying (4) with a sufficiently small bound $\bar{e}$.

Proof:

Since the pair $(A, B)$ is controllable then for any $\delta>0$, there exists a gain $K$ and a matrix $P=P^{T} \succ 0$ such that

$$
A_{c l}^{T} P+P A_{c l} \preceq-2 \delta P,
$$

with $A_{c l}=A+B K$ is Hurwitz. Consider system (1) and the switching law

$$
u(x+e(t)) \in \underset{v \in \mathcal{V}}{\arg \min } \mathcal{G}(x, e(t), v),
$$

with $\mathcal{G}: \mathbb{R}^{n} \times \mathbb{R}^{n} \times \mathcal{V} \rightarrow \mathbb{R}$ defined as $\mathcal{G}(x, e(t), v)=(x+e(t))^{T} \sum_{k=1}^{m}\left(N_{k}(x+e(t))+b_{k}\right) v_{(k)}$. Just as we did in (7) we associate to the closed-loop system (1), (10) a differential inclusion

$$
\dot{x} \in \tilde{\mathcal{F}}(t, x) .
$$

Consider the function $V$ such that $V(x)=x^{T} P x$. We want to prove that there exists $\bar{e}$ such that if (4) is satisfied then

$$
\sup _{y \in \tilde{\mathcal{F}}(t, x)} \frac{\partial V}{\partial x} y \leq-2 \chi V(x),
$$

for some $\chi>0$ in a domain $\mathcal{D} \subset \mathbb{R}^{n}$ which will be determined.

In order to prove these results, we rewrite the system (1) as

$$
\dot{x}=A x+B u+w(x, u),
$$

with $w(x, u)=\sum_{j=1}^{m} N_{j} x u_{(j)}$.

The main idea of the proof of Theorem 1 is to show that for a sufficiently small and bounded perturbation $e$ satisfying (4), the decay of the function $V$ in some domain $\mathcal{D}$ can be ensured by switching among the elements of the set $\mathcal{V}$. This will be proved in the following in three steps. In Step 1, we associate a differential inclusion to system (6) and provide some sufficient conditions for $R \epsilon$-stability. Then, in Step 2, for a decay rate $\delta$ and a static gain $K$ satisfying (9) stability conditions of system 
(13) are given, considering the term $w(x, K x)$ as a perturbation. Finally, in Step 3, based on the results from Steps 1 and 2, we use the property of existence of a static linear stabilizer to design the switching surface such that the Lyapunov function $V$ decreases over the domain of attraction $\mathcal{E}(P, \gamma)$ until its reaches the chattering ball $\mathcal{E}(P, \epsilon)$, which proves the $R \epsilon$-stability of system (1), (10), (4).

\section{Step 1 : Explicit stability condition based on Filippov differential inclusions}

We define for any $z \in \mathbb{R}^{n}$ the set of indexes $\mathcal{I}^{*}(z)$ such that

$$
\mathcal{I}^{*}(z)=\left\{i \in \mathcal{I}_{N}: z^{T} \sum_{k=1}^{m}\left(N_{k} z+b_{k}\right)\left(v_{j(k)}-v_{i(k)}\right) \geq 0, \quad \forall j \in \mathcal{I}_{N}\right\},
$$

which corresponds to the set of minimizers of $u(z)$ defined in (10).

To $\mathcal{I}^{*}(z)$ we associate for all $z \in \mathbb{R}^{n}$ the set $\Delta^{*}(z)$ of vectors defined by

$$
\Delta^{*}(z)=\left\{\beta \in \Delta_{N}: \beta_{(i)}=0, \forall i \in \mathcal{I}_{N} \backslash \mathcal{I}^{*}(z)\right\} .
$$

Using (14) and (15), the set valued map $\tilde{\mathcal{F}}(t, x)$ in (11) satisfies

$$
\tilde{\mathcal{F}}(t, x) \subseteq \tilde{\mathcal{F}}^{*}(t, x),
$$

with

$$
\begin{aligned}
\tilde{\mathcal{F}}^{*}(t, x) & =\operatorname{Conv}_{i \in \mathcal{I}^{*}\left(\bar{x}_{e}(t)\right)}\left\{A x+B v_{i}+w\left(x, v_{i}\right)\right\} \\
& =\left\{A x+B v(\beta)+w(x, v(\beta)): \beta \in \Delta^{*}\left(\bar{x}_{e}(t)\right)\right\},
\end{aligned}
$$

$v(\beta)=\sum_{i=1}^{N} \beta_{(i)} v_{i}$, and $\bar{x}_{e}(t)=x+e(t)$.

Therefore, from (16) and in order to show (12), it is sufficient to prove that for some positive scalar $\chi$ we have

$$
\sup _{y \in \tilde{\mathcal{F}}^{*}(t, x)} \frac{\partial V}{\partial x} y \leq-2 \chi V(x),
$$

in some domain $\mathcal{D} \subset \mathbb{R}^{n}$ to be determined.

From (17), and using the fact that the set $\Delta^{*}(z)$ is compact for all $z \in \mathbb{R}^{n}$, we have

$$
\begin{aligned}
\sup _{y \in \tilde{\mathcal{F}}^{*}(t, x)} \frac{\partial V}{\partial x} y & =\sup _{\beta \in \Delta^{*}\left(\bar{x}_{e}(t)\right)}\left\{\frac{\partial V}{\partial x}(A x+B v(\beta)+w(x, v(\beta)))\right\} \\
& =\max _{\beta \in \Delta^{*}\left(\bar{x}_{e}(t)\right)}\left\{\frac{\partial V}{\partial x}(A x+B v(\beta)+w(x, v(\beta)))\right\} .
\end{aligned}
$$

Then, showing (18) is equivalent to proving that for some $\chi>0$

$$
\max _{\beta \in \Delta^{*}\left(\bar{x}_{e}(t)\right)}\left\{\frac{\partial V}{\partial x}(A x+B v(\beta)+w(x, v(\beta)))\right\} \leq-2 \chi V(x),
$$

in a domain $\mathcal{D} \subset \mathbb{R}^{n}$ to be determined below.

\section{Step 2: Stability properties with a continuous controller}

Here we will study the robustness properties of system (13) with linear state feedback $\bar{u}=K x$ based on the linearized model. These properties will be useful for redesigning a switching controller. Before showing (20), it is useful to prove that

$$
\frac{\partial V}{\partial x}\left(A_{c l} x+w(x, K x)\right) \leq-\delta V(x)
$$

is satisfied in a neighbourhood of the origin where $A_{c l}$ satisfies (9) for some $\delta$ and $P$, and $w(x, K x)=\sum_{j=1}^{m} N_{j} x(K x)_{(j)}$.

From inequality (9), we obtain

$$
\frac{\partial V}{\partial x}\left(A_{c l} x\right) \leq-2 \delta V(x), \forall x \in \mathbb{R}^{n}
$$

Using this, (21) becomes

$$
\frac{\partial V}{\partial x}\left(A_{c l} x+w(x, K x)\right) \leq-2 \delta V(x)+2 x^{T} P w(x, K x), \forall x \in \mathbb{R}^{n},
$$

Furthermore, for some scalar $\varrho>0$ we have

$$
\|w(x, K x)\|=\left\|\sum_{k=1}^{m} N_{k} x(K x)_{(k)}\right\| \leq \varrho\|x\|^{2}, \forall x \in \mathbb{R}^{n} .
$$

Therefore, we can show that

$$
\forall \rho>0, \exists \tilde{\rho}>0:\|w(x, K x)\| \leq \rho\|x\|, \forall\|x\|<\tilde{\rho} .
$$


Then, from Cauchy-Schwarz inequality, we get

$$
x^{T} P w(x, K x) \leq\left\|x^{T} P\right\|\|w(x, K x)\| \leq \rho\|P\|\|x\|^{2}, \forall\|x\|<\tilde{\rho} .
$$

Thus for all $\|x\|<\tilde{\rho}$, we have

$$
\begin{aligned}
\frac{\partial V}{\partial x}\left(A_{c l} x+w(x, K x)\right) & \leq-2 \delta V(x)+2 x^{T} P w(x, K x) \\
& \leq-\left(2 \delta V(x)-2 \rho\|P\|\|x\|^{2}\right) .
\end{aligned}
$$

Therefore, (21) is satisfied for all $x$ such that $\|x\|<\tilde{\rho}$ if we can ensure that

$$
-\left(2 \delta V(x)-2 \rho\|P\|\|x\|^{2}\right) \leq-\delta V(x),
$$

which is verified if

$$
\rho \leq \frac{\delta \lambda_{\min }(P)}{2\|P\|} .
$$

Note that for given $\delta$ and $P$ satisfying inequality (9), $\rho$ can always be chosen as small as possible (this only constrains $x$ in a neighbourhood $\mathcal{B}\left(0, \tilde{\rho}^{2}\right)$ of the origin) such that (24) is verified.

In the next step we will see how the property (24) is useful for the design of a switching law.

\section{Step 3: Switching controller reconfiguration}

Note that, since Assumption A-2 holds, then there exists a neighbourhood of the origin $\mathcal{E}(P, \gamma)$ with $\gamma>0$ such that for all $x \in \mathcal{E}(P, \gamma)$ we have

$$
K x \in \operatorname{Conv}\{\mathcal{V}\} .
$$

Therefore, for all $x \in \mathcal{E}(P, \gamma)$ there exist positive scalars $\alpha_{j}(x), j \in \mathcal{I}_{N}$, such that $\sum_{j=1}^{N} \alpha_{j}(x)=1$ and

$$
K x=\sum_{j=1}^{N} \alpha_{j}(x) v_{j}
$$

In the development that follows we consider that $\mathcal{E}(P, \gamma) \subseteq \mathcal{B}\left(0, \tilde{\rho}^{2}\right)$ (we can always choose a constant $\gamma$ satisfying this inclusion). We can also consider the case where (21) and (25) are verified (i.e. for all $x \in \mathcal{E}(P, \gamma) \subseteq \mathcal{B}\left(0, \tilde{\rho}^{2}\right)$ ). From (14), for all $i \in \mathcal{I}^{*}\left(\bar{x}_{e}(t)\right)$ with $\bar{x}_{e}(t)=x+e(t)$, we have

$$
(x+e(t))^{T} P \sum_{k=1}^{m}\left(N_{k}(x+e(t))+b_{k}\right)\left(v_{j(k)}-v_{i(k)}\right) \geq 0, \forall j \in \mathcal{I}_{N} .
$$

Then, for any $\beta \in \Delta^{*}\left(\bar{x}_{e}(t)\right)$ we have

$$
(x+e(t))^{T} P \sum_{k=1}^{m}\left(N_{k}(x+e(t))+b_{k}\right)\left(v_{j(k)}-v(\beta)_{(k)}\right) \geq 0, \forall j \in \mathcal{I}_{N} .
$$

Multiplying this last inequality by $\alpha_{j}(x)$ for $j \in \mathcal{I}_{N}\left(\alpha_{j}(x)\right.$ defined in (25)) and summing the $N$ elements, we obtain

$$
2(x+e(t))^{T} P \sum_{k=1}^{m}\left(N_{k}(x+e(t))+b_{k}\right)\left((K x)_{(k)}-v(\beta)_{(k)}\right) \geq 0 .
$$

Adding this to the left part of (20), it comes

$$
\begin{aligned}
& \max _{\beta \in \Delta^{*}\left(\bar{x}_{e}(t)\right)}\left\{\frac{\partial V}{\partial x}(A x+B v(\beta)+w(x, v(\beta)))\right\} \\
& \leq \max _{\beta \in \Delta^{*}\left(\bar{x}_{e}(t)\right)}\left\{x^{T}\left(A_{c l}^{T} P+P A_{c l}\right) x+2 x^{T} P w(x, K x)+2 x^{T} P \sum_{k=1}^{m} N_{k} e(t)\left((K x)_{(k)}-v(\beta)_{(k)}\right)\right. \\
& +2 e^{T} P \sum_{k=1}^{m} N_{k} x\left((K x)_{(k)}-v(\beta)_{(k)}\right)+2 e^{T} P \sum_{k=1}^{m} N_{k} e(t)\left((K x)_{(k)}-v(\beta)_{(k)}\right) \\
& \left.+2 e^{T} P B(K x-v(\beta))\right\} .
\end{aligned}
$$

Then, using (21) we get

$$
\begin{aligned}
& \max _{\beta \in \Delta^{*}\left(\bar{x}_{e}(t)\right)}\left\{\frac{\partial V}{\partial x}(A x+B v(\beta)+w(x, v(\beta)))\right\} \\
& \leq \max _{\beta \in \Delta^{*}\left(\bar{x}_{e}(t)\right)}\left\{-\delta V(x)+2 x^{T} P \sum_{k=1}^{m} N_{k} e(t)\left((K x)_{(k)}-v(\beta)_{(k)}\right)+2 e^{T} P B(K x-v(\beta))\right. \\
& \left.+2 e^{T} P \sum_{k=1}^{m} N_{k} x\left((K x)_{(k)}-v(\beta)_{(k)}\right)+2 e^{T} P \sum_{k=1}^{m} N_{k} e(t)\left((K x)_{(k)}-v(\beta)_{(k)}\right)\right\} .
\end{aligned}
$$


Let us define $\bar{v}_{(k)}=\max _{x \in \mathcal{E}(P, \gamma)}\left\{\left(\sum_{j=1}^{N} \alpha_{j}(x) v_{j}\right)_{(k)}-\max _{\beta \in \Delta^{*}\left(\bar{x}_{e}(t)\right)}\left\{v(\beta)_{(k)}\right\}\right\}$. From (25), we have

$$
\max _{\beta \in \Delta^{*}\left(\bar{x}_{e}(t)\right)}\left\{(K x)_{(k)}-v(\beta)_{(k)}\right\}=\max _{\beta \in \Delta^{*}\left(\bar{x}_{e}(t)\right)}\left\{\left(\sum_{j=1}^{N} \alpha_{j}(x) v_{j}\right)_{(k)}-v(\beta)_{(k)}\right\} \leq \bar{v}_{(k)}, \forall k \in \mathcal{I}_{m} .
$$

Thus, using (28), from (27) we obtain that for all $x \in \mathcal{E}(P, \gamma) \subseteq \mathcal{B}\left(0, \tilde{\rho}^{2}\right)$,

$$
\begin{aligned}
& \max _{\beta \in \Delta^{*}\left(\bar{x}_{e}(t)\right)}\left\{\frac{\partial V}{\partial x}(A x+B v(\beta)+w(x, v(\beta)))\right\} \\
& \leq-\delta V(x)+2 x^{T} P \sum_{k=1}^{m} N_{k} \bar{v}_{(k)} e(t)+2 e^{T} P B \bar{v}+2 e^{T} P \sum_{k=1}^{m} N_{k} \bar{v}_{(k)} x+2 e^{T} P \sum_{k=1}^{m} N_{k} \bar{v}_{(k)} e(t) .
\end{aligned}
$$

Recall [8] that for any positive number $\theta$

$$
2 a^{T} b \leq \frac{1}{\theta} a^{T} a+\theta b^{T} b, \forall a, b \in \mathbb{R}^{n}
$$

Applying (30) to the terms $2 e^{T} \Omega x, 2 x^{T} \Omega e$ with $\Omega=P \sum_{k=1}^{m} N_{k} \bar{v}_{(k)}$ and $2 e^{T} P B \bar{v}$, with

$$
\begin{aligned}
& \theta_{1}=\eta, \quad a_{1}=e, \quad b_{1}=\Omega x, \quad \theta_{2}=\eta^{-1}, \quad a_{2}=\Omega^{T} x, \quad b_{2}=e, \\
& \text { and } \theta_{3}=\eta, \quad a_{3}=e, \quad b_{3}=P B \bar{v},
\end{aligned}
$$

we obtain the following inequality

$$
\begin{aligned}
& -\delta V(x)+2 x^{T} P \sum_{k=1}^{m} N_{k} \bar{v}_{(k)} e(t)+2 e^{T} P B \bar{v}+2 e^{T} P \sum_{k=1}^{m} N_{k} \bar{v}_{(k)} x+2 e^{T} P \sum_{k=1}^{m} N_{k} \bar{v}_{(k)} e(t) \\
& \leq-\delta V(x)+3 \eta^{-1} e^{T} e+2 \eta x^{T} \Omega^{T} \Omega x+\eta \bar{v}^{T} B^{T} P P B \bar{v}+2 e^{T} \Omega e .
\end{aligned}
$$

Let us define $\left.\zeta_{\max }=\lambda_{\max }\left(\Omega^{T} \Omega\right) \tilde{\rho}^{2}, \kappa_{\max }=\sqrt{\lambda_{\max }\left(\Omega^{T} \Omega\right.}\right)$, and $\xi_{\max }=\bar{v}^{T} B^{T} P P B \bar{v}$. We have

$$
\begin{gathered}
x^{T} \Omega^{T} \Omega x \leq \zeta_{\max }, \forall x \in \mathcal{B}\left(0, \tilde{\rho}^{2}\right), \\
e^{T} \Omega e \leq \kappa_{\max } e^{T} e, \forall e \in \mathbb{R}^{n} .
\end{gathered}
$$

Then, considering $\bar{e}$ a positive scalar satisfying (4), we obtain from (31)

$$
\begin{aligned}
& -\delta V(x)+2 x^{T} P \sum_{k=1}^{m} N_{k} \bar{v}_{(k)} e(t)+2 e^{T} P B \bar{v}+2 e^{T} P \sum_{k=1}^{m} N_{k} \bar{v}_{(k)} x+2 e^{T} P \sum_{k=1}^{m} N_{k} \bar{v}_{(k)} e(t) \\
& \leq-\delta V(x)+\left(3 \eta^{-1}+\kappa_{\text {max }}\right) \bar{e}+2 \eta \zeta_{\max }+\eta \xi_{\text {max }}
\end{aligned}
$$

for all $x \in \mathcal{E}(P, \gamma) \subseteq \mathcal{B}\left(0, \tilde{\rho}^{2}\right)$.

From (29) and (32), in order to prove (20), it is sufficient to show that there exists $\chi>0$ such that in some domain $\mathcal{D}:=\mathcal{E}(P, \gamma) \backslash \mathcal{E}(P, \epsilon) \subseteq \mathcal{B}\left(0, \tilde{\rho}^{2}\right)$ we have

$$
-\delta V(x)+\left(3 \eta^{-1}+\kappa_{\max }\right) \bar{e}+2 \eta \zeta_{\max }+\eta \xi_{\max } \leq-2 \chi V(x) .
$$

Therefore, we can show that if we take $0<\chi<\frac{\delta}{2}$, and sufficiently small $\bar{e}$ and $\eta$, we have (33) (and thus (12)) which is satisfied for all $x \in \mathcal{E}(P, \gamma) \backslash \mathcal{E}(P, \epsilon) \subseteq \mathcal{B}\left(0, \tilde{\rho}^{2}\right)$, with

$$
\epsilon:=\frac{\left(3 \eta^{-1}+\kappa_{\max }\right) \bar{e}+\eta \xi_{\max }+2 \eta \zeta_{\max }}{\delta-2 \chi}<\gamma
$$

In conclusion, system (1), (10) with perturbation (4) is $R \epsilon$-stable from $\mathcal{E}(P, R)$ to $\mathcal{E}(P, \epsilon)$ with $R=\gamma$ and $\epsilon$ given in (34), if the bound $\bar{e}$ on the perturbation is small enough.

Remark 1: From the proof of Theorem 1, (see equation (34)), we can note that the size of the level set $\mathcal{E}(P, \epsilon)$ depends on the upper bound of the disturbance. Furthermore, it depends also on the upper bound of the control value (the terms $\xi_{\max }$, $\zeta_{\max }$, and $\kappa_{\max }$ depend on $\bar{v}$ defined in (28)). Then, the size of the chattering ball $\mathcal{E}(P, \epsilon)$ increases with the amplitude of the control vector.

In Theorem 1 we have shown that there exists a switching law such that the system is $R \epsilon$-stable for small enough perturbation. In the following, we propose a constructive numerical implementation based in LMIs that allows to design such a controller. 


\section{LMIS FORMALIZATION USING CONTROLLER REDESIGN}

The first result (Theorem 1) has a qualitative nature. As we have seen in the proof of Theorem 1, it is possible to use the property of existence of a linear static feedback to redesign switching surfaces for system (1), (3), (4). In practice, it is useful to find a constructive procedure which, for desired domain of attraction and chattering ball, provides a switching law which ensures the $R \epsilon$-stability. Here we would like to ensure that the domain of attraction contains the ball $\mathcal{B}\left(0, r_{\gamma}\right)$ of radius $\sqrt{r_{\gamma}}$ and that the chattering zone is included in a ball $\mathcal{B}\left(0, r_{c}\right)$ of radius $\sqrt{r_{c}}$. In this section a numerical approach to deal with the design problem is given, ensuring this property. An LMI solution is proposed hereafter. In order to express the result, note that for any finite set of vectors $\mathcal{V}=\left\{v_{1}, \ldots, v_{N}\right\} \subset \mathbb{R}^{m}$, there exists a finite number $n_{h}$ of vectors $h_{i} \in \mathbb{R}^{1 \times m}, i \in \mathcal{I}_{n_{h}}$, such that

$$
\operatorname{Conv}\{\mathcal{V}\}=\left\{u \in \mathbb{R}^{m}: h_{i} u \leq 1, i \in \mathcal{I}_{n_{h}}\right\} .
$$

To provide LMI conditions, we rewrite the system (1) as

$$
\dot{x}=A x+\Pi(u) x+B u,
$$

with $\Pi(u)=\sum_{i=1}^{m} N_{i} u_{(i)}$.

Theorem 2: Assume that A.2 holds. Consider the closed-loop system (1), (3), (4) with $\Gamma=P B, P$ a design parameter, and positive scalars $r_{c}$ and $r_{\gamma}$ such that $r_{c}<r_{\gamma}$ and $\chi>0$. Consider $K$ such that $A_{c l}=A+B K$ is Hurwitz. If there exist $\epsilon_{1}>0, \epsilon_{2}>0, \epsilon_{3}>0, c>0, \gamma>0$ and $P=P^{T} \succ 0$ such that

1)

$$
\mathcal{M}\left(v_{i}\right)=\left[\begin{array}{ccc}
M_{i} & P B K & 0 \\
* & -\epsilon_{3} I & -P B v_{i} \\
* & * & -\psi
\end{array}\right] \preceq 0, \forall i \in \mathcal{I}_{N}
$$

with

$$
\begin{aligned}
M_{i} & =\left(A_{c l}+\Pi\left(v_{i}\right)\right)^{T} P+P\left(A_{c l}+\Pi\left(v_{i}\right)\right)+\left(2 \chi+\epsilon_{1}-\epsilon_{2}\right) P, \\
\psi & =-\epsilon_{1} c+\epsilon_{3} \bar{e}+\epsilon_{2} \gamma,
\end{aligned}
$$

2)

$$
P-K^{T} h_{i}^{T} \gamma h_{i} K \succ 0, \forall i \in \mathcal{I}_{n_{h}}
$$

3)

$$
P \preceq \frac{\gamma}{r_{\gamma}} I,
$$

4)

$$
P \succeq \frac{c}{r_{c}} I
$$

5)

$$
c<\gamma
$$

then the system (1), (3) is $R \epsilon$-stable from $\mathcal{E}(P, \gamma)$ to $\mathcal{E}(P, c)$. Furthermore, $\mathcal{B}\left(0, r_{\gamma}\right) \subseteq \mathcal{E}(P, \gamma)$ and $\mathcal{E}(P, c) \subseteq \mathcal{B}\left(0, r_{c}\right)$.

Proof: We want to prove that if the set of LMIs (36)-(41) is feasible, then the closed-loop system (1), (3) is $R \epsilon$-stable from $\mathcal{E}(P, \gamma)$ to $\mathcal{E}(P, c)$. It is sufficient to prove that the function $V$ defined by $V(x)=x^{T} P x$ satisfies

$$
\sup _{y \in \mathcal{F}^{*}(t, x)} \frac{\partial V}{\partial x} y \leq-2 \chi V(x), \forall x \in \mathcal{E}(P, \gamma) \backslash \mathcal{E}(P, c),
$$

with $\mathcal{F}^{*}(t, x)$ defined in (17).

Let us define the set $\mathcal{C}_{v}$ as

$$
\mathcal{C}_{v}=\left\{x \in \mathbb{R}^{n}: h_{i} K x<1, i \in \mathcal{I}_{N}\right\}
$$

From (38) we have

$$
x^{T} K^{T} h_{i}^{T} h_{i} K x<x^{T} \frac{P}{\gamma} x, \forall i \in \mathcal{I}_{n_{h}}, \forall x \in \mathbb{R}^{n},
$$

and thus, the ellipsoid $\mathcal{E}(P, \gamma)$ satisfies

$$
\mathcal{E}(P, \gamma) \subset \mathcal{C}_{v}
$$

The LMI (36) is equivalent to

$$
z^{T} \mathcal{M}\left(v_{i}\right) z \leq 0, \forall z \in \mathbb{R}^{2 n+1} .
$$

Considering the vector $z^{T}=(x, e, 1)^{T}$, this leads to

$$
\begin{aligned}
& x^{T}\left(\left(A_{c l}+\Pi\left(v_{i}\right)\right)^{T} P+P\left(A_{c l}+\Pi\left(v_{i}\right)\right)\right) x+\left(2 \chi-\epsilon_{2}+\epsilon_{1}\right) x^{T} P x+2 e^{T} P B K x \\
& -2 e^{T} P B v_{i}-\epsilon_{3} e^{T} e-\epsilon_{1} c+\epsilon_{2} \gamma+\epsilon_{3} \bar{e} \leq 0, \forall x \in \mathbb{R}^{n}, \forall e \in \mathbb{R}^{n}, \forall i \in \mathcal{I}_{N} .
\end{aligned}
$$

Note that for all $x \in \mathcal{C}_{v}$, there exist $N$ positive scalars $\alpha_{j}(x), j \in \mathcal{I}_{N}, \sum_{j=1}^{N} \alpha_{j}(x)=1$ such that

$$
K x=\sum_{j=1}^{N} \alpha_{j}(x) v_{j} .
$$


Since the constraint (42) is satisfied, then using (43) and (44), we obtain

$$
\begin{aligned}
& x^{T}\left(\left(A+\Pi\left(v_{i}\right)\right)^{T} P+P\left(A+\Pi\left(v_{i}\right)\right)\right) x+\left(2 \chi-\epsilon_{2}+\epsilon_{1}\right) x^{T} P x+2 e^{T} P B \sum_{j=1}^{N} \alpha_{j}(x) v_{j} \\
& +2 x^{T} P B \sum_{j=1}^{N} \alpha_{j}(x) v_{j}-2 e^{T} P B v_{i}-\epsilon_{3} e^{T} e-\epsilon_{1} c+\epsilon_{2} \gamma+\epsilon_{3} \bar{e} \leq 0, \forall x \in \mathcal{E}(P, \gamma), \forall e \in \mathbb{R}^{n},
\end{aligned}
$$

$\forall i \in \mathcal{I}_{N}$, which leads to

$$
\begin{aligned}
& \sum_{j=1}^{N} \alpha_{j}(x)\left\{2 x^{T} P\left(A+\Pi\left(v_{i}\right)\right) x+2 x^{T} P B v_{j}+\left(2 \chi-\epsilon_{2}+\epsilon_{1}\right) x^{T} P x-\epsilon_{3} e^{T} e+2 e^{T} P B\left(v_{j}-v_{i}\right)\right. \\
& \left.-\epsilon_{1} c+\epsilon_{2} \gamma+\epsilon_{3} \bar{e}\right\} \leq 0, \forall x \in \mathcal{E}(P, \gamma), \forall e \in \mathbb{R}^{n}, \forall i \in \mathcal{I}_{N} .
\end{aligned}
$$

By adding and subtracting the term $2 \sum_{j=1}^{N} \alpha_{j}(x) x^{T} P B\left(v_{j}-v_{i}\right)$, we get

$$
\begin{aligned}
& \sum_{j=1}^{N} \alpha_{j}(x)\left\{2 x^{T} P\left(A+\Pi\left(v_{i}\right)\right) x+2 x^{T} P B v_{i}+\left(2 \chi-\epsilon_{2}+\epsilon_{1}\right) x^{T} P x+2(x+e(t))^{T} P B\left(v_{j}-v_{i}\right)\right. \\
& \left.-\epsilon_{3} e^{T} e-\epsilon_{1} c+\epsilon_{2} \gamma+\epsilon_{3} \bar{e}\right\} \leq 0, \forall x \in \mathcal{E}(P, \gamma), \forall e \in \mathbb{R}^{n}, \forall i \in \mathcal{I}_{N} .
\end{aligned}
$$

For $z \in \mathbb{R}^{n}$, we define $\mathcal{I}^{*}(z)$ and $\Delta^{*}(z)$ as in (14), (15). By construction, we see that

$$
\forall i \in \mathcal{I}^{*}(x+e(t)), \forall j \in \mathcal{I}_{N},(x+e(t))^{T} P B\left(v_{j}-v_{i}\right) \geq 0 .
$$

Furthermore, inequality (41) guarantees the fact that $\mathcal{E}(P, c) \subset \mathcal{E}(P, \gamma)$. Then, for $x \in \mathcal{E}(P, \gamma) \backslash \mathcal{E}(P, c)$, it is clear that $x^{T} P x>c$, and $x^{T} P x \leq \gamma$. Therefore, taking this into account, as well as (46) and the fact that $e^{T} e \leq \bar{e}$ (according to (4)), we can deduce from $(4 \overline{5)}$ that

$$
\frac{\partial V}{\partial x}\left(A x+B v_{i}+\Pi\left(v_{i}\right) x\right) \leq-2 \chi V(x), \forall i \in \mathcal{I}^{*}(x+e(t)), \forall x \in \mathcal{E}(P, \gamma) \backslash \mathcal{E}(P, c) .
$$

Then, using the same arguments as in Theorem 1, we can show that

$$
\sup _{y \in \mathcal{F}(t, x)} \frac{\partial V}{\partial x} y \leq \max _{\beta \in \Delta^{*}\left(\bar{x}_{e}(t)\right)} \frac{\partial V}{\partial x}(A x+B v(\beta)+\Pi(v(\beta)) x) \leq-2 \chi V(x), \forall x \in \mathcal{E}(P, \gamma) \backslash \mathcal{E}(P, c),
$$

with $v(\beta)=\sum_{i=1}^{N} \beta_{(i)} v_{i}$, which ends the proof. Note that the LMI (39) is equivalent to the constraint

$$
\mathcal{B}\left(0, r_{\gamma}\right) \subset \mathcal{E}(P, \gamma)
$$

where $\mathcal{B}\left(0, r_{\gamma}\right)$ is the ball of radius $\sqrt{r_{\gamma}}$, and the LMI (40) is equivalent to the constraint

$$
\mathcal{E}(P, c) \subset \mathcal{B}\left(0, r_{c}\right),
$$

where $\sqrt{r_{c}}$ is the radius of the ball $\mathcal{B}\left(0, r_{c}\right)$. These constraints are used for the optimization of either the chattering ball $\mathcal{E}(P, c)$ or the domain of attraction $\mathcal{E}(P, \gamma)$ (see Remark 2).

Remark 2: The method uses the property of the existence of linear state feedback with gain $K$ in order to design the switching surfaces $\Gamma$. Designing a static gain $K$ for systems as (1) is a classical problem. Numerical methods have been proposed in [1], [44]. To solve the conditions of Theorem 2 as an LMI problem for a given gain $K$ such that $A_{c l}=A+B K$ is Hurwitz $P, c$, and $\gamma$ are taken as LMI variables. The parameter $\chi$ in Theorem 2 corresponds to the system's decay rate from $\mathcal{E}(P, \gamma)$ to $\mathcal{E}(P, c)$. A line search can be used to find $r_{c}$ and $r_{\gamma}$, and parameters $\epsilon_{1}, \epsilon_{2}$, and $\epsilon_{3}$. An optimization algorithm can then be used to maximize $r_{\gamma}$ or minimize $r_{c}$. Note that when the conditions of Theorem 2 are satisfied, the continuous controller $\bar{u}=K x$ ensures a decay rate of at least $2 \chi+\epsilon_{1}-\epsilon_{2}$. In this paper we only provide sufficient conditions for the design of a switching controller while the measurements are affected by perturbation. The proposed conditions are not necessary. What we show is that, if the LMIs are satisfied for a given gain $K$ and a decay rate $\chi$, then a locally stabilizing switching controller is obtained with guaranteed estimations of the domain of attraction and chattering zone. The approach is based on the inclusion $\tilde{\mathcal{F}}(t, x) \subseteq \tilde{\mathcal{F}}^{*}(t, x)$ (equations (16), (17) used in (47)) which might be a source of conservatism. The polytopic modelling of the bilinear term can also introduce some conservatism.

In Sections III and IV, stability conditions of the closed-loop system have been given. In order to show the efficiency of the developed method, numerical implementations have been performed. The results are reported in the next section.

\section{NUMERICAL EXAMPLES}

\section{A. Example 1: Single-input system}

Consider the switched affine system (2) with

$$
\tilde{A}_{1}=\left[\begin{array}{cc}
0.3 & 1 \\
1 & 0.3
\end{array}\right], \quad \tilde{A}_{2}=\left[\begin{array}{cc}
-0.1 & 1 \\
-\frac{1}{3} & -0.1
\end{array}\right], \quad \tilde{b}_{1}=\left[\begin{array}{c}
1.5 \\
6
\end{array}\right], \quad \tilde{b}_{2}=\left[\begin{array}{c}
-0.5 \\
1
\end{array}\right], \quad \text { and } \quad \sigma=\{1,2\} .
$$

Considering the result in [23], this system can be reformulated as a bilinear system (1) with 


$$
u \in \mathcal{V}=\left\{v_{1}, v_{2}\right\}=\{0.75,-0.25\}, \quad N=\left[\begin{array}{cc}
0.4 & 0 \\
1.3333 & 0.4
\end{array}\right], \quad A=\left[\begin{array}{ll}
0 & 1 \\
0 & 0
\end{array}\right], \quad \text { and } \quad B=\left[\begin{array}{l}
2 \\
5
\end{array}\right] .
$$

Note that the proposed methods in the literature can not be used to stabilize the system since there exist no convex combination of the matrices $A_{1}$ and $A_{2}$. We consider a gain $K=\left[\begin{array}{ll}-0.4, & -0.44\end{array}\right]$ computed by pole assignment such that the eigenvalues of $A+B K$ are $\{-1,-2\}(A+B K$ is Hurwitz) and a desired decay rate $\chi=0.15$. Using the results from Theorem 2, we can design a switching law (3) that makes the system (1), (3), (4) $R \epsilon$-stable for a given bound $\bar{e}$ on the perturbation. Then, implementing an optimization algorithm as discussed in Remark 2 allows the minimization of the ball $\mathcal{B}\left(0, r_{c}\right)$ containing the chattering zone. The results obtained for various values of $\bar{e}$ are given in Table I. For $\bar{e}=10^{-4}$ for example, we find that the LMIs from Theorem 2 are feasible for

$$
r_{\gamma}=1.5, \quad r_{c}=0.14, \quad \gamma=0.0589, \quad c=0.0027, \quad P=\left[\begin{array}{cc}
0.03 & 0.0091 \\
0.0091 & 0.03
\end{array}\right],
$$

and with the parameters $\epsilon_{1}=0.71, \epsilon_{2}=0$, and $\epsilon_{3}=1.1980 \times 10^{2}$. Simulations are performed for different initial conditions $x(0)=\left[\begin{array}{ll}-0.92, & 1.28\end{array}\right]^{T}, x(0)=\left[\begin{array}{ll}-0.12, & 1.4\end{array}\right]^{T}, x(0)=\left[\begin{array}{ll}-1.28, & -0.08\end{array}\right]^{T}, x(0)=\left[\begin{array}{ll}0.32, & -1.4\end{array}\right]^{T}, x(0)=$ $\left[\begin{array}{ll}1.12, & -1.08\end{array}\right]^{T}, x(0)=\left[\begin{array}{ll}1.36, & -0.12\end{array}\right]^{T}, x(0)=\left[\begin{array}{ll}0.84, & 0.84\end{array}\right]^{T}, x(0)=\left[\begin{array}{ll}-0.68, & -0.96\end{array}\right]^{T}$ and a perturbation $e(t)=$ $\sqrt{\bar{e}}[\sin (3 t), \quad \cos (3 t)]^{T}$. The different trajectories are reported in the phase plot given in Figure 1, while the state variables starting at $x(0)=\left[\begin{array}{ll}-0.92,1.28\end{array}\right]^{T}$ are presented in Figure 2.

Using the above design method, a switching law is derived and the domain of attraction is successfully estimated. From the simulation results, it can be seen that for a sufficiently small perturbation, the states variables starting in the domain of attraction $\mathcal{E}(P, \gamma)$ converge to a small neighbourhood $\mathcal{E}(P, c)$ of the origin and oscillate indefinitely around it. This confirms the theoretical results. Note that, in Figure 1 is reported the sliding surface of the system free of perturbation $\left(x^{T} \Gamma=0\right)$. We can remark that the sliding surface in the presence of the perturbation is variable since its depends on the perturbation dynamic and variations $\left((x+e)^{T} \Gamma=0\right)$.

TABLE I

CHATTERING BALL RADIUS $r_{c}$ OBTAINED FOR DIFFERENT VALUES OF $\bar{e}$

\begin{tabular}{|l|l|l|l|l|l|l|}
\hline $\bar{e}$ & $5 \times 10^{-5}$ & $10^{-4}$ & $5 \times 10^{-4}$ & $10^{-3}$ & $5 \times 10^{-3}$ & $10^{-2}$ \\
\hline$r_{c}$ & 0.1 & 0.14 & 0.55 & 0.69 & 1.1 & 1.4 \\
\hline
\end{tabular}

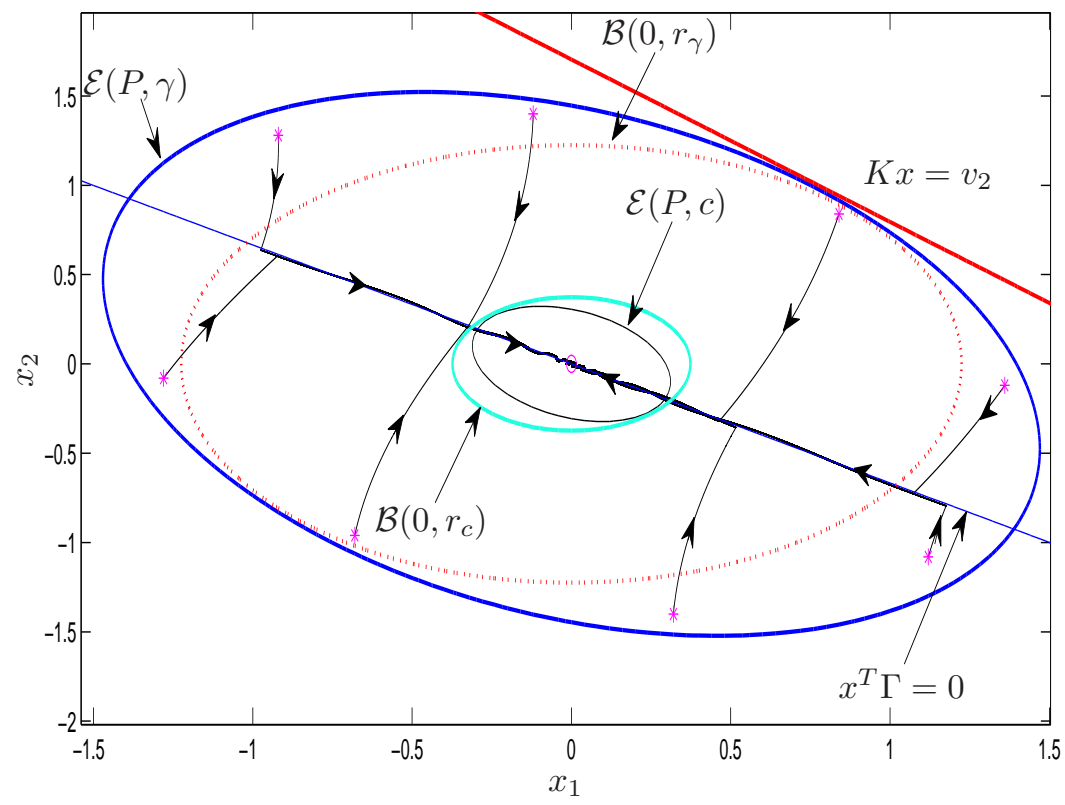

Fig. 1. Phase portrait of the closed-loop system with $\mathcal{E}(P, \gamma)$ an estimation of the domain of attraction, and $\mathcal{E}(P, c)$ the chattering zone. 


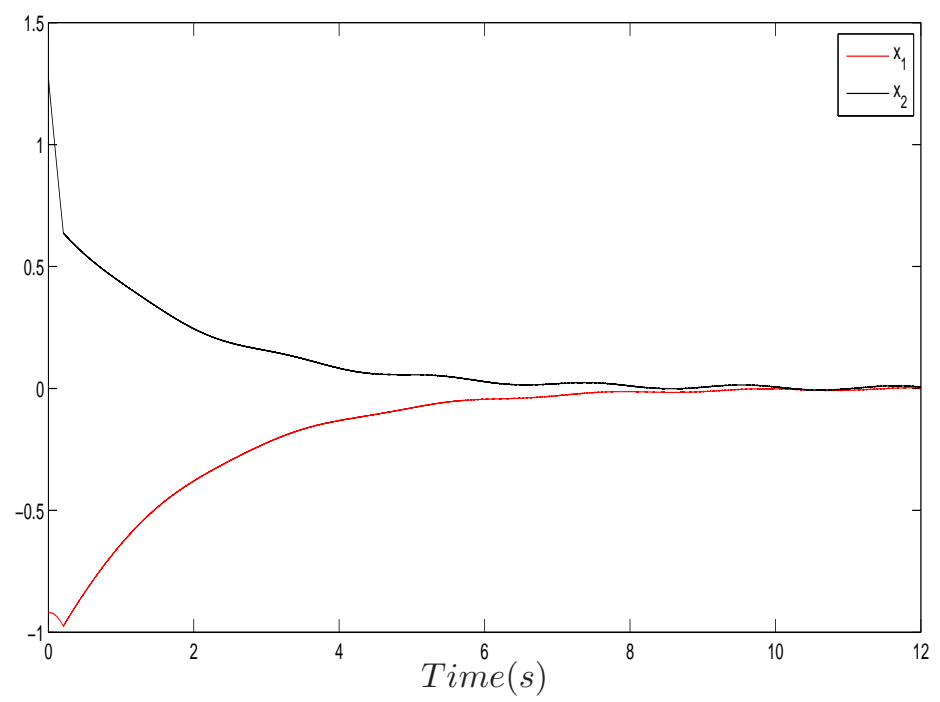

Fig. 2. State variables $x_{1}$ and $x_{2}$

\section{B. Example 2: Multi-input system}

Consider system (1) with

$$
\begin{aligned}
& A=\left[\begin{array}{cc}
8 & 1.5 \\
1.5 & -3
\end{array}\right], \quad B=\left[\begin{array}{cc}
8 & -3 \\
2 & 1
\end{array}\right], N_{1}=\left[\begin{array}{cc}
0 & 0 \\
0.1 & 0.2
\end{array}\right], N_{2}=\left[\begin{array}{cc}
0 & 0 \\
0 & 0.2
\end{array}\right], \\
& \text { and } u \in \mathcal{V}=\left\{\left[\begin{array}{l}
5 \\
5
\end{array}\right],\left[\begin{array}{c}
5 \\
-5
\end{array}\right],\left[\begin{array}{c}
-5 \\
5
\end{array}\right],\left[\begin{array}{l}
-5 \\
-5
\end{array}\right]\right\} .
\end{aligned}
$$

The eigenvalues of $A$ are $\{-3.2,8.2\}$ hence the open-loop system (1) is unstable. Note the requirement from [13] is not satisfied since the matrix $A$ is not Hurwitz. Therefore, the existing methods in the literature can not be used to stabilize the system. Consider the gain

$$
K=\left[\begin{array}{cc}
-1.0714 & -0.6429 \\
0.6429 & -1.2143
\end{array}\right]
$$

such that the matrix $A+B K$ is Hurwitz (the gain $K$ is computed by pole assignment such that the eigenvalues of $A+B K$ are $\{-5.5,-2.5\}$ ). Applying the method developed above with a decay rate $\chi=2$, a switching law is designed to stabilize the system in the presence of a perturbation bounded by $\bar{e}=10^{-4}$. An algorithm of minimization of $r_{c}$ with a line search to find the parameters $\epsilon_{1}, \epsilon_{2}, \epsilon_{3}, r_{\gamma}$ and $r_{c}$ is implemented.

Using our algorithm of optimization we find that the LMIs are feasible for

$$
r_{\gamma}=13.2, \quad r_{c}=1.6, \quad \gamma=0.2728, \quad c=0.0324, \quad P=\left[\begin{array}{cc}
0.02 & -0.0001 \\
-0.0001 & 0.021
\end{array}\right],
$$

and with parameters $\epsilon_{1}=0.7250, \epsilon_{2}=0$, and $\epsilon_{3}=1.1980 \times 10^{2}$.

Simulations are performed for different initial conditions $x(0)=\left[\begin{array}{ll}1.3, & 3.2\end{array}\right]^{T}, x(0)=\left[\begin{array}{ll}-3.4, & -0.8\end{array}\right]^{T}, x(0)=$ $\left[\begin{array}{ll}0, & 3.5\end{array}\right]^{T}, x(0)=\left[\begin{array}{ll}3, & 1.5\end{array}\right]^{T}, x(0)=\left[\begin{array}{ll}3.5, & -0.1\end{array}\right]^{T}, x(0)=\left[\begin{array}{ll}-3.1, & 1.4\end{array}\right]^{T}, x(0)=\left[\begin{array}{ll}3.4, & 0.9\end{array}\right]^{T}, x(0)=\left[\begin{array}{ll}-3.5, & 0.1\end{array}\right]^{T}$, $x(0)=\left[\begin{array}{ll}-3, & 2\end{array}\right]^{T}, x(0)=\left[\begin{array}{ll}-1.7, & 3\end{array}\right]^{T}, x(0)=\left[\begin{array}{cc}1.9, & -0.6\end{array}\right]^{T}, x(0)=\left[\begin{array}{ll}1.74, & 2\end{array}\right]^{T}, x(0)=\left[\begin{array}{ll}1.5, & -3.1\end{array}\right]^{T}, x(0)=$ $\left[\begin{array}{ll}-2.97,-1.9536\end{array}\right]^{T}, x(0)=[2.83, \quad-1.93]^{T}$, and a perturbation $e(t)=\sqrt{\bar{e}}[\sin (3 t), \quad \cos (3 t)]^{T}$. The different trajectories are reported in the phase plot given in Figure 4, while the state variables starting at $x(0)=\left[\begin{array}{ll}3.4, & 0.9\end{array}\right]^{T}$ are presented in Figure 3. 


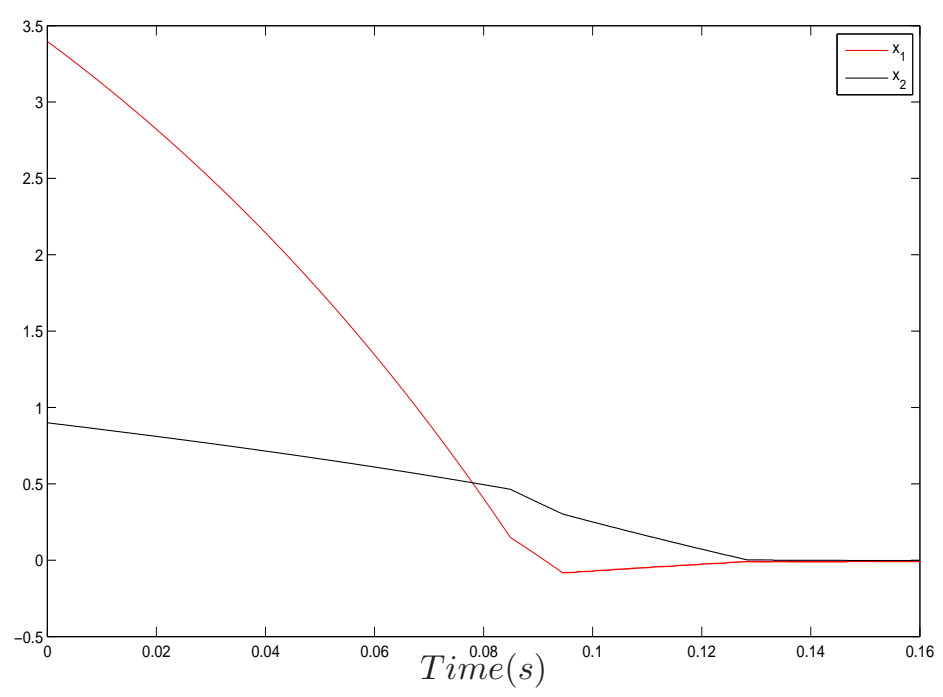

Fig. 3. State variables $x_{1}$ and $x_{2}$

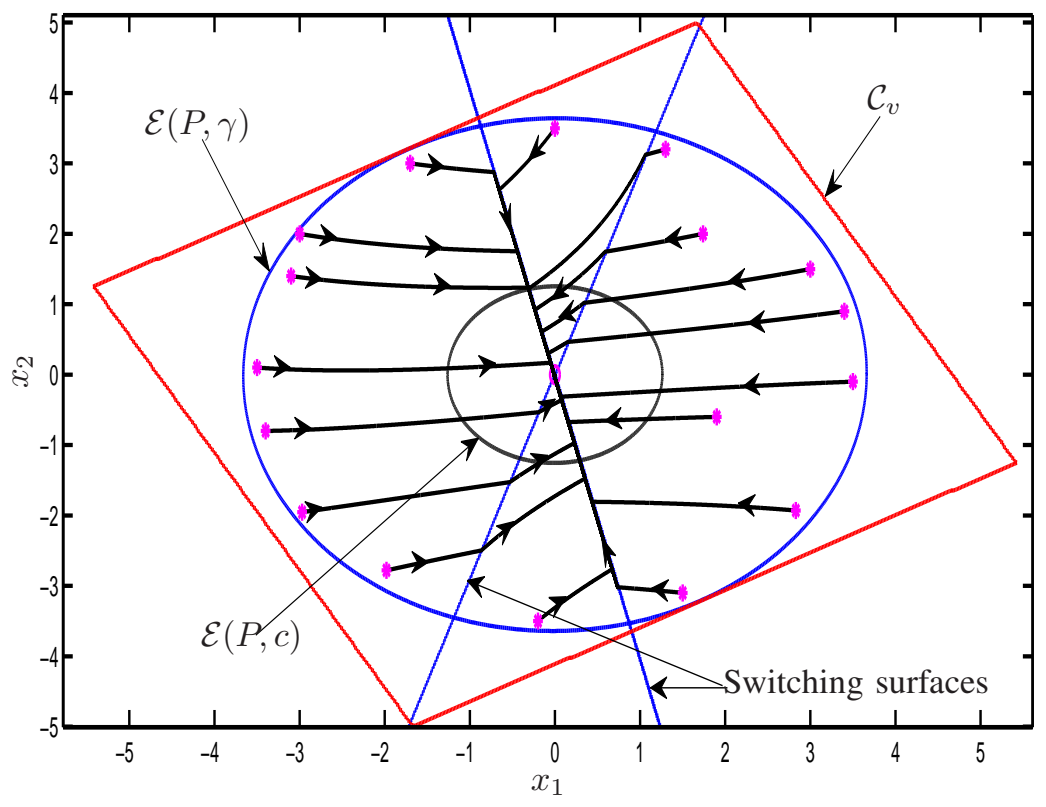

Fig. 4. Phase portrait of the closed-loop system with $\mathcal{E}(P, \gamma)$ an estimation of the domain of attraction, and $\mathcal{E}(P, c)$ the chattering zone.

\section{CONCLUSION}

This paper has provided a method for the stabilization of switched affine systems with state-dependent switching laws. The method considers the perturbation in the states measurements. Qualitative conditions for stability are developed. In addition, a numerical approach allowing a simple numerical implementation of the designed method is given. Computer simulations are presented to illustrate the efficiency of the given method. In the future, the approach may be improved in various manners. For example, it is of interest to considerer the bilinear terms in the switching law design method. In order to reduce the chattering, we can try to extend the approach to the case of min-switching stategy with min-dwell time condition. In this direction we can follow the approach in [24] or the one proposed in [11]. The study of the output dependent switching law design and observer based controller may be envisaged in future works.

\section{REFERENCES}

[1] V. Andrieu and S. Tarbouriech. Global asymptotic stabilization for a class of bilinear systems by hybrid output feedback. Transactions on Automatic Control, 58(6):1602-1608, 2013. 
[2] P. J. Antsaklis. Hybrid control systems: An introductory discussion to the special issue. Transactions on Automatic Control, 43(4):457-460, 1998.

[3] J. P. Aubin and A. Cellina. Differential inclusions: set-valued maps and viability theory. 1984.

[4] A. Bacciotti and L. Rosier. Liapunov functions and stability in control theory. Springer Science \& Business Media, 2006.

[5] A. G. Beccuti, S. Mariéthoz, S. Cliquennois, S. Wang, and M. Morari. Explicit model predictive control of DC-DC switched-mode power supplies with extended Kalman filtering. Transactions on Industrial Electronics, 56(6):1864-1874, 2009.

[6] S. C. Bengea and R. A. DeCarlo. Optimal control of switching systems. Automatica, 41(1):11-27, 2005.

[7] P. Bolzern and W. Spinelli. Quadratic stabilization of a switched affine system about a nonequilibrium point. In American Control Conference, volume 5, pages 3890-3895. IEEE, 2004.

[8] S. P. Boyd, L. El Ghaoui, E. Feron, and V. Balakrishnan. Linear matrix inequalities in system and control theory, volume 15. SIAM, 1994.

[9] M. S. Branicky. Multiple Lyapunov functions and other analysis tools for switched and hybrid systems. Transactions on Automatic Control, 43(4):475-482, 1998.

[10] J. Cortes. Discontinuous dynamical systems. Control Systems, 28(3):36-73, 2008.

[11] G. S. Deaecto, M. Souza, and J. C. Geromel. Chattering free control of continuous-time switched linear systems. IET Control Theory \& Applications, 8(5): 348-354, 2014.

[12] G. S. Deaecto, J. C. Geromel, and J. Daafouz. Dynamic output feedback $H_{\infty}$ control of switched linear systems. Automatica, 47(8): 1713-1720, 2011.

[13] G. S. Deaecto, J. C. Geromel, F.S. Garcia, and J.A. Pomilio. Switched affine systems control design with application to DC-DC converters. IET Control Theory \& Applications, 4(7):1201-1210, 2010.

[14] D. L. Elliott. Bilinear control systems: matrices in action, volume 169. Springer Science \& Business Media, 2009.

[15] A. F. Filippov and F. M. Arscott. Differential equations with discontinuous righthand sides: control systems, volume 18. Springer Science \& Business Media, 1988

[16] C. Fiter and E. Fridman. Stability of piecewise affine systems with state-dependent delay, and application to congestion control. In 52nd Conference on Decision and Control, pages 1572-1577. IEEE, 2013.

[17] B. Gerard, H. Souley Ali, M. Zasadzinski, and M. Darouach. $H_{\infty}$ filter for bilinear systems using LPV approach. Transactions on Automatic Control, 55(7):1668-1674, 2010.

[18] J. C. Geromel, and P. Colaneri. Stability and stabilization of continuous-time switched linear systems. SIAM Journal on Control and Optimization, 2006, vol. 45, no 5, p. 1915-1930.

[19] R. Goebel, R. G. Sanfelice, and A. R. Teel. Hybrid Dynamical Systems: modeling, stability, and robustness. Princeton University Press, 2012.

[20] J. M. Gonçalves, A. Megretski, M. Dahleh, et al. Global stability of relay feedback systems. Transactions on Automatic Control, 46(4):550-562, 2001.

[21] S. Govindaswamy, L. Hetel, A. Polyakov, and T. Floquet. On relay control for discrete time systems using linear matrix inequalities. In European Control Conference, pages 2225-2230. IEEE, 2014.

[22] P. Hauroigné, P. Riedinger, and C. Iung. Switched affine systems using sampled-data controllers: robust and guaranteed stabilization. Transactions on Automatic Control, 56(12):2929-2935, November 2011.

[23] L. Hetel and E. Bernuau. Local stabilization of switched affine systems. Transactions on Automatic Control, 60(4):1158-1163, 2015.

[24] L. Hetel and E. Fridman. Robust sampleddata control of switched affine systems. Transactions on Automatic Control, 58(11): 2922-2928, 2013.

[25] L. Hetel, M. Defoort, and M. Djemai. Binary control design for a class of bilinear systems: Application to a multilevel power converter. Transactions on Control Systems Technology, PP(99):1-1, 2015.

[26] T. Hu and F. Blanchini. Non-conservative matrix inequality conditions for stability/stabilizability of linear differential inclusions. Automatica,46(1),190-196, 2010.

[27] K. H. Johansson. Relay feedback and multivariable control. PhD thesis, Lund University, 1997.

[28] K. H. Johansson, A. Rantzer, and K. J. strm. Fast switches in relay feedback systems, 1999.

[29] M. Jungers, C. C. Gonzaga, and J. Daafouz. Min-switching local stabilization for discrete-time switching systems with nonlinear modes. Nonlinear Analysis: Hybrid Systems, 2013, vol. 9, p. 18-26.

[30] B.-S. Kim and M.-T. Lim. Robust $H_{\infty}$ control method for bilinear systems. International Journal of Control, Automation, and Systems,1(2):171-177, 2003.

[31] D. Liberzon. Switching in systems and control. Springer Science \& Business Media, 2003.

[32] H. Lin and P. J. Antsaklis. Stability and stabilizability of switched linear systems: a survey of recent results. Transactions on Automatic control, 54(2):308-322, 2009.

[33] T. Liu and F. Gao. Industrial process identification and control design: step-test and relay-experiment-based methods. Springer Science \& Business Media, 2011.

[34] D. Patino, P. Riedinger, and C. Iung. Practical optimal state feedback control law for continuous-time switched affine systems with cyclic steady state. International Journal of Control, 82(7):1357-1376, 2009.

[35] S. Pettersson. Synthesis of switched linear systems. In 42nd Conference on Decision and Control, volume 5, pages 5283-5288. IEEE, 2003.

[36] S. Pettersson, and B. Lennartson. Stabilization of hybrid systems using a min-projection strategy. In IEEE Proceedings of the 2001 American Control Conference, pp. 223-228.

[37] A.E. Polyakov. On practical stabilization of systems with delayed relay control. Automation and Remote Control, 71(11):2331-2344, 2010.

[38] A. Poznyak, A. Polyakov, and V. Azhmyakov. Attractive Ellipsoids in Robust Control. Springer, 2014.

[39] A. Rantzer and M. Johansson. Piecewise linear quadratic optimal control. Transactions on Automatic Control, 45(4):629-637, 2000.

[40] P. Riedinger and I.-C. Morarescu. A numerical framework for optimal control of switched affine systems with state constraint. In 4th IFAC Conference on Analysis and Design of Hybrid Systems, pages 141-146, 2012.

[41] C. Seatzu, D. Corona, A. Giua, and A. Bemporad. Optimal control of continuous-time switched affine systems. Transactions on Automatic Control, 51(5):726-741, 2006.

[42] H. Sira-Ramírez and R. Silva-Ortigoza. Control design techniques in power electronics devices. Springer Science \& Business Media, 2006.

[43] S. Tarbouriech and G. Garcia. Control of uncertain systems with bounded inputs. Springer-Verlag New York, Inc., 1997.

[44] S. Tarbouriech, I. Queinnec, T. R. Calliero, and P.L.D. Peres. Control design for bilinear systems with a guaranteed region of stability: An lmi-based approach. In 17th Mediterranean Conference on Control and Automation, pages 809-814. IEEE, 2009.

[45] A. Trofino, D. Assmann, C. C. Scharlau, and D. F. Coutinho. Switching rule design for switched dynamic systems with affine vector fields. Transactions on Automatic Control, 54(9):2215-2222, 2009. 
[46] A. Trofino, C. C. Scharlau, T. J.M. Dezuo, and D. O. Mauricio C. Stabilizing switching rule design for affine switched systems. In 50th Conference on Decision and Control and European Control Conference, pages 1183-1188. IEEE, 2011.

[47] A. Trofino, C. C. Scharlau, Tiago J.M. Dezuo, and D. O. Mauricio C. Switching rule design for affine switched systems with performance. In 51st Conference on Decision and Control, pages 1923-1928. IEEE, 2012. 